\title{
Differential Diagnostic Approaches in Traditional Chinese Medicine and Western Physiotherapy for Mental Illness and Psychosocial Disorders: Similarities and Challenges in Integrating and Promoting East-Meet-West Approaches - Mini Review
}

\section{Wong HY and Law YM*}

Department of Rehabilitation Sciences, Hong Kong Polytechnic University, Hong Kong

*Corresponding author: Law Ying Man, Department of Rehabilitation Sciences, Hong Kong Polytechnic University, Hong Kong, Tel: 852-2766-6724;

Email: ymlaw.law@polyu.edu.hk

\section{Mini Review \\ Volume 2 Issue 1}

Received Date: January 10, 2019

Published Date: January 21, 2019

DOI: $10.23880 /$ aphot-16000115

\section{Abstract}

The improvement of accurate management of psychosocial disorders and mental illness are the raising concern in the field of rehabilitation profession in the recent decade. The combination of the features and values of traditional Chinese medicine (TCM) and western physiotherapy approaches has also been advocated worldwide. It remains unclear for the integration and implementation of TCM and western approaches for promoting more accurate diagnosis and management of mental illness and psychosocial disorders from a rehabilitation perspective. This article provides a minioverview about the similarities and challenges about eastern and western approaches for the rehabilitation needs of mental illness and psychosocial disorders in clinical practice. This may facilitate further discussion and improvement of combining the values of eastern and western healthcare concepts and methods for the benefits of the people with various psychosocial and mental health needs.

Keywords: Chinese Medicine; Diagnosis; Physiotherapy; Psychosocial; Mental

Abbreviations: TCM: Traditional Chinese Medicine; WHO: World Health Organization

\section{Introduction}

According to the World Health Organization (WHO), psychosocial disorders, especially depressions is one of the main causes of disability worldwide and are estimated to be affecting 300 million people globally. It shows that psychosocial disorder is quite an alarming issue affecting the globe $[1,2]$. From the WHO, psychosocial disorders lead to a large economic consequence with an estimated lost economic output of US $\$ 16.3$ million between 2011 and 2030. Therefore, it is necessary to put more focus on the improvement of current management of psychosocial and mental health rehabilitation, concerning to the growing healthcare and health economic burdens of these health problems [1,2].

Psychosocial disorder and/or mental illness can be the consequences of multiple factors including but not limited to social, genetic, physiological, environmental, mental, 


\section{Annals of Physiotherapy \& Occupational Therapy}

cultural and physical factors. From the angle of Traditional Chinese Medicine (TCM), it disturbs balance of Yin and Yang, Qi and Blood, causing behavioral or psychological symptoms, which affects multiple areas of life of the individual. An accurate diagnosis is the key of success in practice. It guides the development of our professions [3]. Physiotherapists make physical diagnosis different from the medical diagnosis made by the doctor but similar to the differential diagnosis made by the traditional TCM practitioners.

To make an accurate diagnosis, apart from the professional examination techniques, clinical reasoning also plays a major role. Many studies discussed the challenges faced by physiotherapists in diagnostic reasoning with most of them focusing on the education levels. This article aims at discussing the similarities and challenges of differential diagnosis used by TCM practitioners and physiotherapists as well as raising the concern about the practical problems, especially in the management of mental illness and psychosocial disorders. The key focus of this paper is on similarities and challenges in relation to TCM and western physiotherapy approaches for the needs of mental illness and psychosocial disorders.

\section{Similarities and Benefits}

TCM uses pattern differentiation or called syndrome differentiation as the basic diagnosis of disease. It is an accurate diagnosis with treatment based on different syndromes and it helps the integration of basic theories and clinical practice. In TCM, diagnosis was made based on the clinical characteristics of patients and then a specific treatment plan was created based on the differential diagnosis. Syndrome is a combination of TCM signs and symptoms with disease diagnosis, environment and patient profile. Take depression, the most common psychosocial disorder, as example, TCM states that depression is caused by imbalance of different organs such as liver, spleen, heart or gas and blood. As a result, it should be diagnosed based on the development of disease for specific patients and patients would be prescribed with different kinds of drugs.

Similarly, physiotherapists make physical diagnosis based on subjective examination which includes the body chart, behavior of symptoms, history and the objective examination which includes observation, palpation and active and passive movement as well as some special tests. Instead of looking purely into the pathology of the diseases for a medical diagnosis, both of the two diagnostic methods are based on identification of clusters of signs and symptoms of patients with other related information. For example, patients with low back pain can have different clinical presentation and high clinical reasoning skills are required to differentiate the underlying sources [4]. As a result, clinical reasoning skills and differential diagnosis are essential in providing relevant treatment to patients.

With the aid of differential diagnosis, professionals can classify the same disease into different sub-groups and design an integrated treatment plan with the improved understandings of the specificity and complexity of the disorders. As a result, patients can receive a tailor-made treatment.

\section{Challenges in Research}

Comparing to TCM, physiotherapy has a stronger evidence base. However, an interesting phenomenon is that there were increasing evidences showing the effectiveness of treatment but lacks evidences in guiding therapists to select treatment based on physical diagnosis. Evidences of TCM were of a lower level due to the problems in standardization. Many researches about TCM did not show clearly the intervention method or included a combination of intervention in one study because of the uniqueness of TCM intervention. To satisfy criterion of high evidence, researches about physiotherapy was usually based on patients group and provided them with the standardized treatment plan.

However, it can only show the effectiveness of the treatment on the patients group. It cannot provide evidence on how to guide us to select the best treatment plan for the patients based on differential diagnosis in the clinical practice. As a result, further research can be done to fill in the gap between research and clinical practice.

\section{Challenges in Clinical Practice}

Differential diagnosis can help with designing a specific treatment plan. However, such diagnostic method can also lead to many difficulties in clinical practice. Concerning pattern differentiation of TCM, practitioners have to first gather information on the presenting signs and symptoms and then analyze the information using the TCM theory of pathogenesis or etiology. In the procedure of making an accurate diagnosis among all different types of syndrome of one disease, clinical experiences of the practitioners are crucial. A research showed that the experimental group which was prescribed with Chinese 


\section{Annals of Physiotherapy \& Occupational Therapy}

medicine based on syndrome-based differential diagnosis had significantly better treatment effects and lacked complication when compared with the control group.

However, it included only 4 types of syndrome of depression whereas there are more than 10 types. As a result, in evaluating the clinical significance of TCM in treating psychosocial disorder, the method of diagnosis and the related challenges faced in practice should also be considered. A review article of TCM concerning lateral elbow pain, a condition commonly seen in physiotherapy practice, has summarized 16 patterns based on 40 common or unique signs and symptoms [5]. Apart from showing the complication of the diagnosis, the author also suggested validating and standardizing pattern differentiation by the use of biological markers as well as grouping similar patterns to help in the ease of clinical application.

Concerning physiotherapy practice, increasing number of protocols were developed in guiding treatment plan for the ease of clinical practice. However, patients may not be able to receive the best treatment that is unique and most beneficial to their problems. As a result, efforts should be made in balancing the ease of practice and the benefits of patients with such practice. For the development of physiotherapy practice, guidelines and summaries of differential diagnosis can be made.

\section{Implications}

Psychosocial disorder is common diseases that affect people's mental and physical health and increasing financial burden of the medical system. Both western and eastern medicines advocate 'prevention is a better medicine than cure', this review article provides an overview of the similarities of diagnostic methods of TCM and physiotherapy in relation to the management of psychosocial disorders and mental illness. We can take references on approaches of TCM.

Studies on TCM are seeking for evidences to support their theories of diagnosis whereas studies on physiotherapy has a trend of increasing focus on the practical issue like generalization, effectiveness and ease of practice. There are still gaps between research and clinical practice. Differential diagnosis is undoubtedly challenging in clinical practice and required clinical reasoning skills. As a result, we have to balance its benefits and its feasibility in clinical practice with regards to the unique values of eastern and western approaches.
In the modern medical and health system, there is an increase in the number of studies and practitioners advocate the mutual referral system between western and eastern medicine in practice. The topic of psychosocial disorder and mental illness share similarities between western and eastern medicine. This can act as a precursor for the fusion of the two types of medicine. After all, we should consider benefits on patients as our final goal.

Studies are still needed to systematic identify and manage the determinants, facilitators and barriers for promoting the integration and application of TCM and western rehabilitation concepts and methods for the improvement of quality of the management of psychosocial disorders and mental illness in the future.

\section{Conclusion}

In conclusion, full understanding about effective integration of the features of west and east differential diagnostic approaches for the management of psychosocial disorders and mental illness remain incomplete. Individuals with mental illness or psychosocial disorders are unique but still sharing common clinical signs and symptoms. Whilst some studies discussed various differential diagnostic approaches in traditional Chinese medicine and western physiotherapy, many factors are still needed to be considered in practice to correctly analyse and interpret the clinical presentations and findings obtained from eastern and western differential diagnoses. This may improve the clinical management of the rehabilitation for different mental illness and psychosocial factors using the values from eastern and western concepts. Research is still needed to identify and manage the determinants affecting the integration and implementation of eastern and western approaches for specific types of mental illness and psychosocial disorders.

\section{References}

1. WHO (2017) Scalable psychological interventions.

2. WHO (2013) Mental health action plan 2013-2020.

3. Sahrmann SA (1988) Diagnosis by the physical therapist-a prerequisite for treatment: a special communication. Phys Ther 68(11): 1703-1706.

4. Karvonen E, Paatelma M, Laitinen-Vaananen S, Piirainen A (2017) Clinical reasoning and critical 


\section{Annals of Physiotherapy \& Occupational Therapy}

reflection in physiotherapists' examinations of patients with low back pain in its early phase: a qualitative study from physiotherapists' point of view. European journal of physiotherapy 19(4): 185193.
5. Gadau M, Zhang SP, Yip HY, Yeung WF, Bian Z X, et al. (2016) Pattern Differentiation of Lateral Elbow Pain in Traditional Chinese Medicine: A Systematic Review. J Altern Complement Med 22(11): 921-935. 